\title{
MCTS1 promotes the development of lung adenocarcinoma by regulating E2F1 expression
}

\author{
CUN GAO, RUI DONG*, YONGMENG LI", JINGHUI LIANG* ${ }^{*}$ and HUI TIAN \\ Department of Thoracic Surgery, Qilu Hospital, Cheeloo College of Medicine, \\ Shandong University, Jinan, Shandong 250012, P.R. China
}

Received September 15, 2020; Accepted April 16, 2021

DOI: 10.3892/ol.2021.12792

\begin{abstract}
Lung adenocarcinoma (LUAD) is the most common subtype of lung cancer that results in the majority of cancer-associated mortality. Multiple copies in T-cell lymphoma-1 (MCTS1) is an oncogene that is expressed at high levels in several types of cancer tissues. However, its exact role and pathomechanism in the development of LUAD remains unknown. Reverse transcription-quantitative PCR analysis was performed to detect MCTS1 expression. Immunohistochemistry analysis was performed to detect MCTS1 expression in LUAD tissues and normal tissues. The MTT, colony formation, EdU, flow cytometry, wound healing and Transwell assays were performed to assess the proliferation, apoptosis, migration and invasion of LUAD cells. Western blot analysis was performed to detect protein expression levels. The present study aimed to investigate the effects of MCTS1 on the progression of LUAD and the potential mechanisms underlying its effects. The results demonstrated that MCTS1 expression was upregulated in LUAD tissues and cells, which was associated with an unfavorable outcome in patients with LUAD. MCTS1 knockdown inhibited LUAD progression by suppressing cell viability and motility, and promoting apoptosis. In addition, E2F1 protein expression was attenuated following MCTS1 knockdown. The silencing MCTS1-induced inhibitory effect on LUAD malignancy was reversed following overexpression of E2F1 by modulating the c-Myc signaling pathway. Taken together, the results of the present study suggest that MCTS1 facilitates cell proliferation and migration, and suppresses apoptosis of LUAD cells by regulating E2F1 expression and the c-Myc signaling pathway.
\end{abstract}

Correspondence to: Dr Hui Tian, Department of Thoracic Surgery, Qilu Hospital, Cheeloo College of Medicine, Shandong University, 44 Wenhuaxi Road, Jinan, Shandong 250012, P.R. China

E-mail: tianhuiql@126.com

${ }^{*}$ Contributed equally

Key words: multiple copies in T-cell lymphoma-1 lung adenocarcinoma, E2F1, proliferation, apoptosis

\section{Introduction}

Lung cancer is the most common cancer and the leading cause of cancer-associated mortality worldwide $(1,2)$. The majority of patients with lung cancer are diagnosed during advanced stages (3). However, for patients diagnosed at early stages and who receive surgical resection or definitive chemotherapy, the recurrence rate is as high as $90 \%$ (3). Lung adenocarcinoma (LUAD) is the most common subtype of lung cancer $(4,5)$. Currently, increasing evidence suggest that the prognosis of patients with LUAD is far from ideal, with a 5-year survival rate of only $15 \%$ (6). Thus, it is important to identify the effective markers for diagnosis and novel therapeutic targets of LUAD.

Multiple copies in T-cell lymphoma-1 (MCTS1), also known as MCT-1, is an oncogene that was initially identified to be upregulated in T-cell lymphoma (7). Increasing evidence suggest that targeting MCTS1 can promote genomic instability and tumorigenesis in different types of cancer (8-10). MCTS1 is associated with lymphomageneses, including the role of stimulation of cell proliferation and angiogenesis, and inhibition of apoptosis (11). Overexpression of MCTS1 attenuates cell doubling time, shortens the period of $\mathrm{G}_{1}$ transition and promotes the expression of cyclin D1 in diffuse large B-cell lymphomas (12). Weng et al (13) reported that MCTS1 expression is a novel prognostic marker in breast cancer, and upregulation of MCTS1 promotes epithelial-to-mesenchymal transition (EMT) and activates matrix metalloproteinase in triple-negative breast cancer cell. Notably, upregulated MCTS1 expression accelerates the progression of lung cancer by regulating the YY1-EGFR-MnSOD signaling pathway (13). In addition, MCTS1 mediating IL-6/Stat3 signaling promotes the stemness of non-small cell lung cancer (NSCLC) cells (8). However, the role of MCTS1 in LUAD remains largely unknown.

The E2F family is composed of E2F1-3a and E2F3b, 4-8, which is predominantly regulated by the retinoblastoma family and plays a key role in various cellular behaviors $(14,15)$. E2F1 is the most extensively studied transcription factor in different types of human cancer (16-20). It contains conserved DNA binding domains and through these putative domains, E2F1 can closely bind to the promoter of target genes and modulate the expression of genes participating in the cell cycle (21). E2F1 has also been identified as a key transcription factor 
in NSCLC (22). However, the association between E2F1 and MCTS1 in LUAD remains unknown.

The present study aimed to investigate the effects of MCTS1 on the progression of LUAD and the potential mechanisms underlying its effects. The results of the present study demonstrated that MCTS1 functions as an oncogene in the development of LUAD. MCTS1 expression was upregulated in patients with LUAD, which was associated with unfavorable prognosis. Mechanistically, MCTS1 knockdown notably suppressed LUAD cell viability and motility. The potential pathomechanism between MCTS1 and E2F1 in LUAD progression was also investigated. Taken together, the results of the present study suggest that MCTS1 may be used as a prospective biomarker to predict the therapeutic outcomes for patients with LUAD.

\section{Materials and methods}

Tumor tissue samples. The expression of MCTS1 in LUAD tissues was analyzed using the UALCAN cancer OMICs database (http://ualcan.path.uab.edu/index.html) from The Cancer Genome Atlas Lung Adenocarcinoma (TCGA-LUAD) cohort, including 59 normal tissues and 515 tumor tissues. Patients with sufficient follow-up information were included in the overall survival analysis, using the Gene Expression Profiling Interactive Analysis (GEPIA2, http://gepia2.cancer-pku.cn) database.

Tumor tissues and paired peripheral normal lung tissues ( $2 \mathrm{~cm}$ away from the tumor margin) were collected from 30 patients with LUAD (18 men and 12 women; age range, 28-79 years; median age, 53.5 years) who underwent resection surgery at Qilu Hospital of Shandong University between January 2018 and December 2019. The inclusion criteria were as follows: i) None of the patients received neoadjuvant therapy; ii) study patients were diagnosed with LUAD by histopathology and iii) diagnosis was confirmed by two independent pathologists. Complete clinicopathological data for the 30 paired LUAD samples were available upon request. The present study was approved and supervised by the Medical Ethics Committee of Qilu Hospital of Shandong University (Jinan, China; approval no. KYLL-2016-097) and written informed consent was provided by all patients prior to the study start.

Cell lines and small interfering (si)RNA transfection. The human LUAD cell lines, H1573, H23, H1299, A549, H1703 and H1915, and the normal lung cell line, IMR90, were purchased from the American Type Culture Collection and maintained in RPMI-1640 medium (Gibco; Thermo Fisher Scientific, Inc.) supplemented with $10 \%$ fetal bovine serum and $1 \%$ penicillin and streptomycin sulphate (both Hyclone; Cytiva), at $37^{\circ} \mathrm{C}$ with $5 \% \mathrm{CO}_{2}$. The $\mathrm{H} 1573$ cell line was authenticated via short tandem repeat profiling to eliminate cross-contamination.

siRNAs (Guangzhou RiboBio Co., Ltd.) were used to knockdown MCTS1 expression. pcDNA3.1 vector (Shanghai GenePharma Co., Ltd.) was used to overexpress E2F1 expression in LUAD cells (H1573 and H1299). si-MCTS1-1, si-MCTS1-2, negative control siRNA (si-NC), pcDNA3.1-E2F1 and pcDNA3.1-NC were designed and synthesized by Shanghai GenePharma Co., Ltd. The following sequences were used: siMCTS1-1 forward, 5'-CCCUAAGAUUACUUC ACAATT-3' and reverse, 5'-UUGUGA AGUAAUCUUAGG CTT-3'; and siMCTS1-2 forward, 5'-GCAAUUUCCAGG UAUUGAATT-3' and reverse, 5'-UUCAAUACCUGCAAA UUGCTT-3'. For siRNA and pcDNA3.1 transfection, cells were seeded into 6-well plates at a density of $1 \times 10^{6}$ cells/well and incubated for $24 \mathrm{~h}$ at $37^{\circ} \mathrm{C}$. LUAD cells were transfected with siRNAs $(5 \mathrm{nM})$ or pcDNA3.1 vector $(50 \mathrm{nM})$ using Lipofectamine ${ }^{\circledR} 3000$ (Invitrogen; Thermo Fisher Scientific, Inc.), according to the manufacturer's protocol. Transfection efficiency was assessed via reverse-transcription quantitative (RT-q)PCR analysis $48 \mathrm{~h}$ post-transfection.

MTT assay. A total of $1 \times 10^{4} \mathrm{H} 1573$ and $\mathrm{H} 1299$ cells were seeded into a 96-well plate and incubated for $24 \mathrm{~h}$ at room temperature. When the cells reached $80 \%$ confluence, $10 \mu \mathrm{l}$ MTT reagent $(5 \mathrm{mg} / \mathrm{ml}$; Beijing Solarbio Science \& Technology Co., Ltd.) was added to each well and cells were incubated for an additional $2 \mathrm{~h}$ with dimethyl sulfoxide (Beijing Solarbio Science \& Technology Co., Ltd.). Cell proliferation was analyzed at a wavelength of $490 \mathrm{~nm}$ under a spectrophotometer (Thermo Fisher Scientific, Inc.).

EdU assay. H1573 and $\mathrm{H} 1299$ cells were initially incubated with $50 \mu \mathrm{M}$ EdU solution (cat. no. ab222421; Abcam) at $37^{\circ} \mathrm{C}$ for $2 \mathrm{~h}$. Cells were subsequently fixed in $4 \%$ paraformaldehyde at $37^{\circ} \mathrm{C}$ for $30 \mathrm{~min}$ and treated with $0.5 \%$ Triton X-100 (Sigma-Aldrich; Merck KGaA) at $37^{\circ} \mathrm{C}$ for $15 \mathrm{~min}$. Cells were washed twice with PBS and $100 \mu 1$ Hoechst 33342 stain (cat. no. ab228551; Abcam) was subsequently added to each well for $30 \mathrm{~min}$ at $37^{\circ} \mathrm{C}$. EdU positive cells were observed under a fluorescence microscope (Olympus Corporation, magnification, x100).

Colony formation assay. Tumor cells (H1573 and H1299) were seeded into 6-well plates at a density of 500 cells/well and cultured at $37^{\circ} \mathrm{C}$ with $5 \% \mathrm{CO}_{2}$ for 2 weeks. Cell colonies were fixed in $4 \%$ paraformaldehyde at $37^{\circ} \mathrm{C}$ for $20 \mathrm{~min}$ and subsequently stained with $0.1 \%$ crystal violet (Sigma-Aldrich; Merck KGaA) at $37^{\circ} \mathrm{C}$ for $10 \mathrm{~min}$. Cell colonies were manually counted using a stereomicroscope (Thermo Fisher Scientific, Inc., magnification, $\mathrm{x} 40$ ).

Transwell migration assay. A total of $1 \times 10^{5} \mathrm{H} 1573$ and $\mathrm{H} 1299$ cells were plated in the upper chambers of Transwell plates in $200 \mu \mathrm{l}$ RPMI-1640 serum-free medium (Gibco; Thermo Fisher Scientific, Inc.). RPMI-1640 medium (600 $\mu 1$, Gibco; Thermo Fisher Scientific, Inc.) was plated in the lower chambers. Following incubation for $48 \mathrm{~h}$ at $37^{\circ} \mathrm{C}$, the migratory cells were fixed in $4 \%$ paraformaldehyde for $20 \mathrm{~min}$ at $37^{\circ} \mathrm{C}$ and subsequently stained with $0.1 \%$ crystal violet at $37^{\circ} \mathrm{C}$ for $10 \mathrm{~min}$. Stained cells were manually counted in five randomly selected fields using a stereomicroscope (magnification, x200; Thermo Fisher Scientific, Inc.).

Wound healing assay. The wound healing assay was performed to assess cell migration. Cells (H1573 and H1299) were seeded into a 6-well plate for $24 \mathrm{~h}$ at $37^{\circ} \mathrm{C}$ using serum-free medium. Once the cells reached $\sim 80 \%$ confluence (or more) the monolayers were scratched using a sterile $200 \mu$ l pipette tip. Cells 
were washed with PBS to remove debris and the attached cells were cultured for an additional $48 \mathrm{~h}$. The wounded areas were observed under an inverted light microscope (Olympus Corporation, magnification, x100).

Flow cytometric analysis. Cell apoptosis was detected via flow cytometric analysis, using propidium iodide (PI) and Annexin V staining (Invitrogen; Thermo Fisher Scientific, Inc.). Briefly, transfected cells (H1573 and H1299) were harvested and resuspended in pre-cooled PBS buffer. Cells were centrifuged $\left(300 \mathrm{x} \mathrm{g}\right.$ for $5 \mathrm{~min}$ at $\left.37^{\circ} \mathrm{C}\right)$ and resuspended in binding buffer ( $200 \mu 1$, Invitrogen; Thermo Fisher Scientific, Inc.), and incubated with $5 \mu \mathrm{l}$ Annexin V-FITC and PI at room temperature for $15 \mathrm{~min}$ in the dark. Apoptotic cells were subsequently analyzed using the FACS Scan (BD Biosciences).

$R T-q P C R$. Total RNA was extracted from H1573, H23, H1299, A549, H1703, H1915, and IMR90 cells using either TRIzol $^{\circledR}$ reagent (Invitrogen; Thermo Fisher Scientific, Inc.) or the RNeasy Mini kit (Qiagen, Inc). Total RNA was reverse transcribed into cDNA using the PrimeScript ${ }^{\mathrm{TM}} \mathrm{RT}$ reagent kit (Takara Bio, Inc.). RT was performed at $16^{\circ} \mathrm{C}$ for $30 \mathrm{~min}$, $42^{\circ} \mathrm{C}$ for $30 \mathrm{~min}$ and $85^{\circ} \mathrm{C}$ for 5 min. qPCR was subsequently performed using the SYBR Green PCR Master Mix (Takara Bio, Inc.) on the ABI 7900 system. The following thermocycling conditions were used for qPCR: $95^{\circ} \mathrm{C}$ for $10 \mathrm{~min}$ followed by 40 cycles at $95^{\circ} \mathrm{C}$ for $10 \mathrm{sec}, 60^{\circ} \mathrm{C}$ for $20 \mathrm{sec}$ and $72^{\circ} \mathrm{C}$ for $30 \mathrm{sec}$. The following primer sequences were used for qPCR: MCTS1 forward, 5'-TTCCTCGTGTGAGGGGATCT-3' and reverse, 5'-ATAGAAAATCGGCCCCTGCT-3'; E2F1 forward, 5'-GTGCTCTCACCGTCCTACAC-3' and reverse, 5'-CTGCACTTTCGGCCCTTTTG-3'; and GAPDH forward, 5'-GCACCGTCAAGGCTGAGAAC-3' and reverse, 5'-ATG GTGGTGAAGACGCCAGT-3'. Relative expression levels were calculated using the $2^{-\Delta \Delta \mathrm{Cq}}$ method (23) and normalized to the internal reference gene GAPDH.

Western blotting. Total protein was extracted from H1573, H23, H1299, A549, H1703, H1915, and IMR90 cells using RIPA buffer (Thermo Fisher Scientific, Inc.). Total protein was quantified using the BCA Protein Assay kit (Thermo Fisher Scientific, Inc.) and $15 \mu \mathrm{g}$ protein/lane was separated by SDS-PAGE on a $10 \%$ gel. The separated proteins were subsequently transferred onto PVDF membranes (Thermo Fisher Scientific, Inc.) and blocked with $5 \%$ non-fat milk at $37^{\circ} \mathrm{C}$ for $1 \mathrm{~h}$ in the dark. The membranes were incubated with primary antibodies against MCTS1 (cat. no. ab102678), E-cadherin (cat. no. ab227639), N-cadherin (cat. no. ab76011), Vimentin (cat. no. ab92547), B-cell lymphoma 2 (Bcl-2, cat. no. ab182858), Bax (cat. no. ab32503), E2F1 (cat. no. ab4070) and GAPDH (cat. no. ab181602) overnight at $4^{\circ} \mathrm{C}$ (all 1:1,000 and purchased from Abcam). Following the primary incubation, membranes were incubated with secondary antibodies (cat. no. ab7090; 1:1,000; Abcam) for $1 \mathrm{~h}$ at room temperature. Protein bands were visualized using the ECL Western Blotting kit (Thermo Fisher Scientific, Inc.) and analyzed using Image Lab ${ }^{\mathrm{TM}}$ software (version 3.0; Bio-Rad Laboratories, Inc.).

Immunohistochemistry $(I H C)$. Tissue samples were fixed in $10 \%$ neutral buffered formalin (Thermo Fisher Scientific,
Inc.) at $37^{\circ} \mathrm{C}$ for $24 \mathrm{~h}$, dehydrated and embedded in paraffin. Tissue sections were incubated in $3 \% \mathrm{H}_{2} \mathrm{O}_{2}$ for $15 \mathrm{~min}$ at room temperature and blocked in $10 \%$ normal goat serum (Sigma-Aldrich; Merck KGaA) for $30 \mathrm{~min}$ at room temperature. Paraffin-embedded tissue samples were cut into $5-\mu \mathrm{m}$-thick sections. Following dewaxing and rinsing, sections were heated in sodium citrate buffer to retrieve the antigen and endogenous peroxidase activity was quenched. Tissue sections were incubated with primary antibody against MCTS1 (1:1,000; Abcam; cat. no. ab238825) overnight at $4^{\circ} \mathrm{C}$. Following the primary incubation, the sections were incubated with secondary antibody (1:1,000; Abcam; cat. no. ab150077) at room temperature for $30 \mathrm{~min}$. The slides were subsequently stained with DAB (Sigma-Aldrich; Merck KGaA) at room temperature for $30 \mathrm{~min}$ and counterstained with $0.02 \%$ hematoxylin (Sigma-Aldrich; Merck KGaA) at room temperature for $30 \mathrm{sec}$, and observed under a stereomicroscope (magnification, x200; Thermo Fisher Scientific, Inc.).

Bioinformatics analysis. Data on MCTS1 expression in patients with LUAD was retrieved from TCGA data portal (http://cancergenome.nih.gov). UALCAN was used (http://ualcan.path.uab.edu/index.html) to plot the figures of genes expression. Overall survival analysis of patients with LUAD, based on MCTS1 expression, was performed using GEPIA (http://gepia2.cancer-pku.cn). Patients with LUAD were divided into high (MCTS1 expression $>$ median, $\mathrm{n}=238$ ) and low (MCTS1 expression $\leq$ median, $\mathrm{n}=239$ ) MCTS1 expression groups. Gene Set Enrichment Analysis (GSEA) (24) was performed on TCGA database dataset (LUAD; https://cancergenome.nih.gov/) of MCTS1 expression using R package clusterProfiler (25). For GSEA, MCTS1 expression was treated as a numeric variable. The Pearson correlation coefficient of other genes and MCTS1 expression was calculated, and then the genes were sequenced according to the correlation coefficient. Using the hallmark gene sets deposited in the GSEA Molecular Signatures Database resource (h.all.v7.1.symbols.gmt, https://www.gsea-msigdb. org/gsea/index.jsp), the differential pathways between the high-MCTS1 expression and low-MCTS1 expression specimens were identified. The number of permutations was 1,000. NES (normalized enrichment score) $>1$ and FDR (false discovery rate) q-val $<0.05$ were set as cut-offs for significant enrichment.

Statistical analysis. Statistical analysis was performed using GraphPad Prism 7.0 software (GraphPad Software, Inc.) and SPSS 22.0 software (IBM Corp.). All experiments were performed in triplicate and data are presented as the mean \pm standard deviation. Survival analysis was performed using the Kaplan-Meier method and log-rank test. The $\chi^{2}$ test was used to analyze the association between MCTS1 expression and the clinicopathological characteristics of patients with LUAD. A two-tailed paired Student's t-test was used to determine statistical differences in MCTS1 expression between LUAD tissues and matched adjacent normal tissues. Unpaired Student's t-test was used to compare differences between two groups, while one-way ANOVA and Tukey's post hoc test were used to compare differences between multiple groups. $\mathrm{P}<0.05$ was considered to indicate a statistically significant difference. 


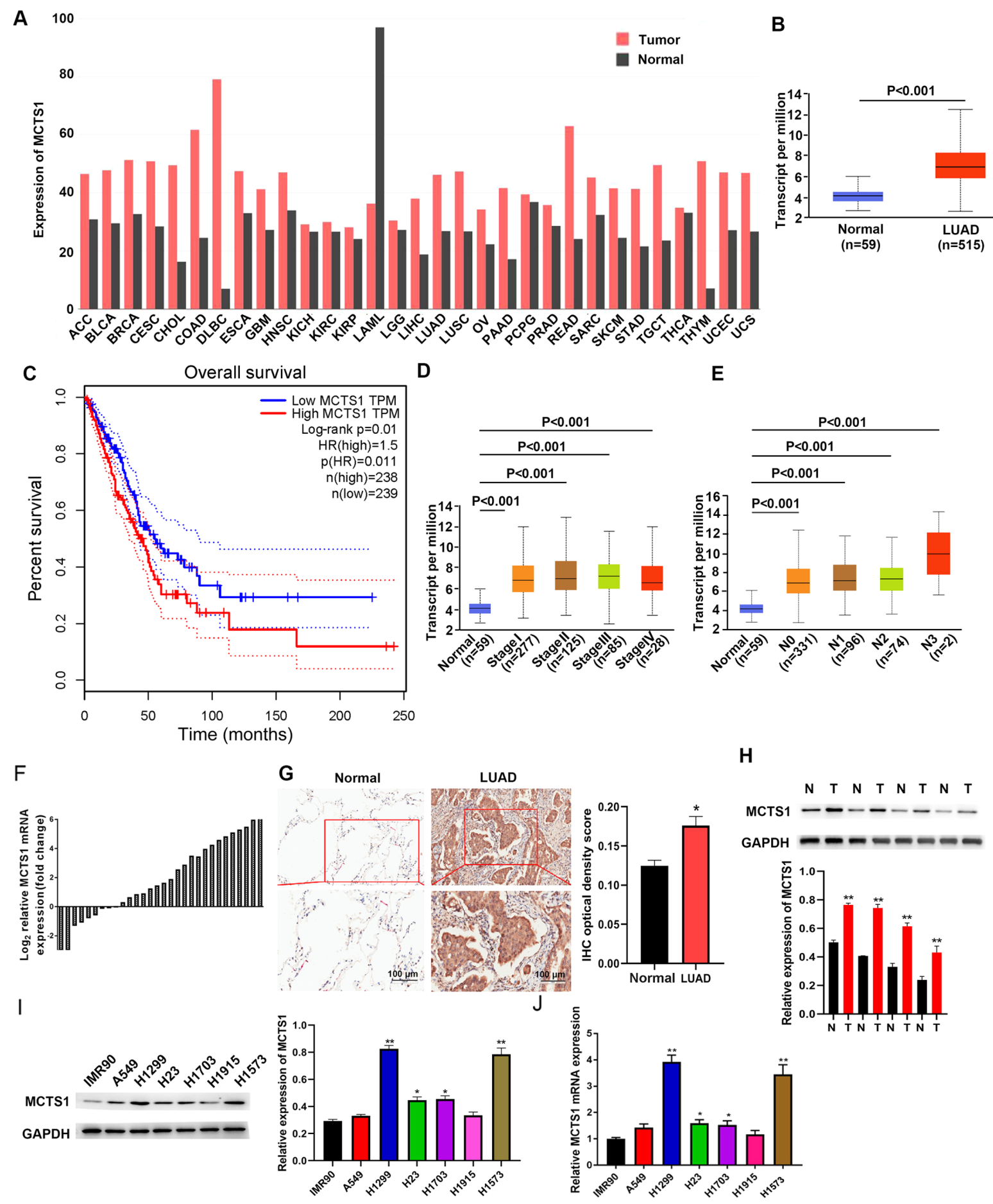

Figure 1. MCTS1 expression is upregulated in LUAD tissues, and closely associated with prognosis and clinicopathological characteristics. (A) MCTS1 expression in TCGA database and comparisons with other tissues. (B) MCTS1 expression in healthy controls and LUAD tissues was detected based on TCGA database. (C) High MCTS1 expression was associated with unfavorable outcomes in patients with LUAD. MCTS1 was expressed at high levels along the LUAD (D) stages and (E) metastasis. (F) RT-qPCR analysis demonstrated that MCTS1 mRNA expression was upregulated in LUAD tissues. (G) Representative MCTS1 immunostaining in healthy and LUAD samples. (H) Western blot analysis demonstrated that MCTS1 protein expression was upregulated in LUAD tissues compared with normal tissues. (I) Western blot and (J) RT-qPCR analyses were performed to detect MCTS1 protein expression in different LUAD cell line. ${ }^{*} \mathrm{P}<0.05,{ }^{* *} \mathrm{P}<0.01$ vs. normal or IMR90 cells. MCTS1, multiple copies in T-cell lymphoma-1; LUAD, lung adenocarcinoma; TCGA, The Cancer Genome Atlas; RT-qPCR, reverse transcription-quantitative PCR; IHC, immunohistochemistry; N, normal; T, tumor.

\section{Results}

MCTS1 expression is upregulated in LUAD, which is associated with unfavorable prognosis. TCGA database was used to determine whether MCTS1 was aberrantly expressed in LUAD tissues compared with normal tissues. As presented in Fig. 1A, MCTS1 expression was upregulated in LUAD tissues compared with normal tissues. To predict the prognostic value of MCTS1, patients with LUAD were classified into two groups, the high and low MCTS1 expression groups, based on the mean value (cut-off value $=0.5, P=0.01$, Fig. $1 B$ ). Survival analysis demonstrated that patients with high 

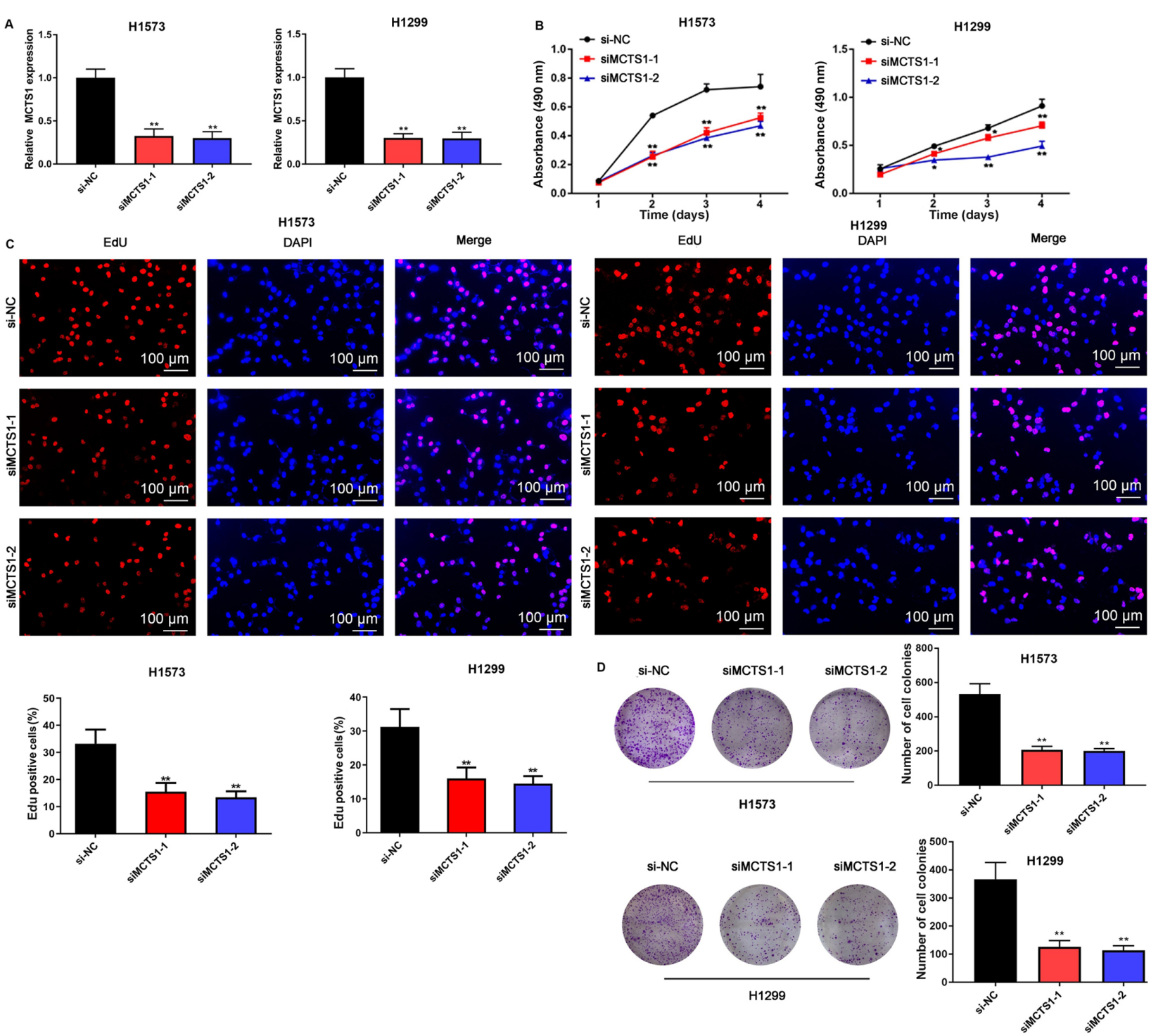

Figure 2. MCTS1 knockdown inhibits the proliferation of lung adenocarcinoma cells. (A) MCTS1 expression significantly decreased in H1573 and H1299 cells following transfection with siMCTS1-1 and siMCTS1-2. The colony forming and proliferative abilities of H1573 and H1299 cells were assessed via the (B) MTT, (C) EdU and (D) colony formation assays. ${ }^{*} \mathrm{P}<0.05,{ }^{* *} \mathrm{P}<0.01 \mathrm{vs}$. si-NC. MCTS1, multiple copies in T-cell lymphoma-1; si, small interfering; $\mathrm{NC}$, negative control.

MCTS1 expression had a shorter overall survival time, while low MCTS1 expression was associated with better prognosis (Fig. 1C). Taken together, these results suggest that MCTS1 may be involved in the progression of LUAD, and highlight the need to investigate its cellular mechanisms.

The association between MCTS1 expression and clinicopathological characteristics was analyzed using profiles of patients with LUAD downloaded from TCGA database. As presented in Fig. 1D, MCTS1 expression was upregulated in LUAD tissues of patients at different stages. Furthermore, MCTS1 expression was upregulated in patients with different node metastasis status compared with the healthy controls (Fig. 1E). Taken together, these results suggest that MCTS1 expression is significantly associated with stage and node metastasis in LUAD.

RT-qPCR and IHC analyses validated upregulated MCTS1 expression in LUAD samples (Fig. 1F and G). Similarly, tissue samples resected from 30 patients with LUAD exhibited high MCTS1 expression compared with adjacent normal tissues (Fig. 1H). High MCTS1 expression levels were detected in H1573 and H1299 cells compared with the other cell lines (A549, H1703, H23 and H1915) and IMR90 cells (Fig. 1I and J). Thus, H1573 and H1299 cells were selected for subsequent experimentation.

\section{MCTS1 knockdown inhibits cell proliferation, migration and} invasion, and increases the apoptotic rate in LUAD cancers. To assess the effect of MCTS1 in LUAD, an in vitro model of MCTS1 knockdown was established and the changes in cellular behaviors were investigated. The results demonstrated that transfection with siMCTS1-1/siMCTS1-2 significantly attenuated MCTS1 mRNA expression in both H1573 and H1299 cells (Fig. 2A). The MTT, EdU, colony formation, wound healing, Transwell and flow cytometry assays were subsequently performed to determine whether aberrant MCTS1 expression affects LUAD cellular malignant behaviors. The results of the MTT assay demonstrated that transfection with siMCTS1-1/siMCTS1-2 markedly inhibited the proliferative ability of H1573 and H1299 cells (Fig. 2B). Similarly, the results 

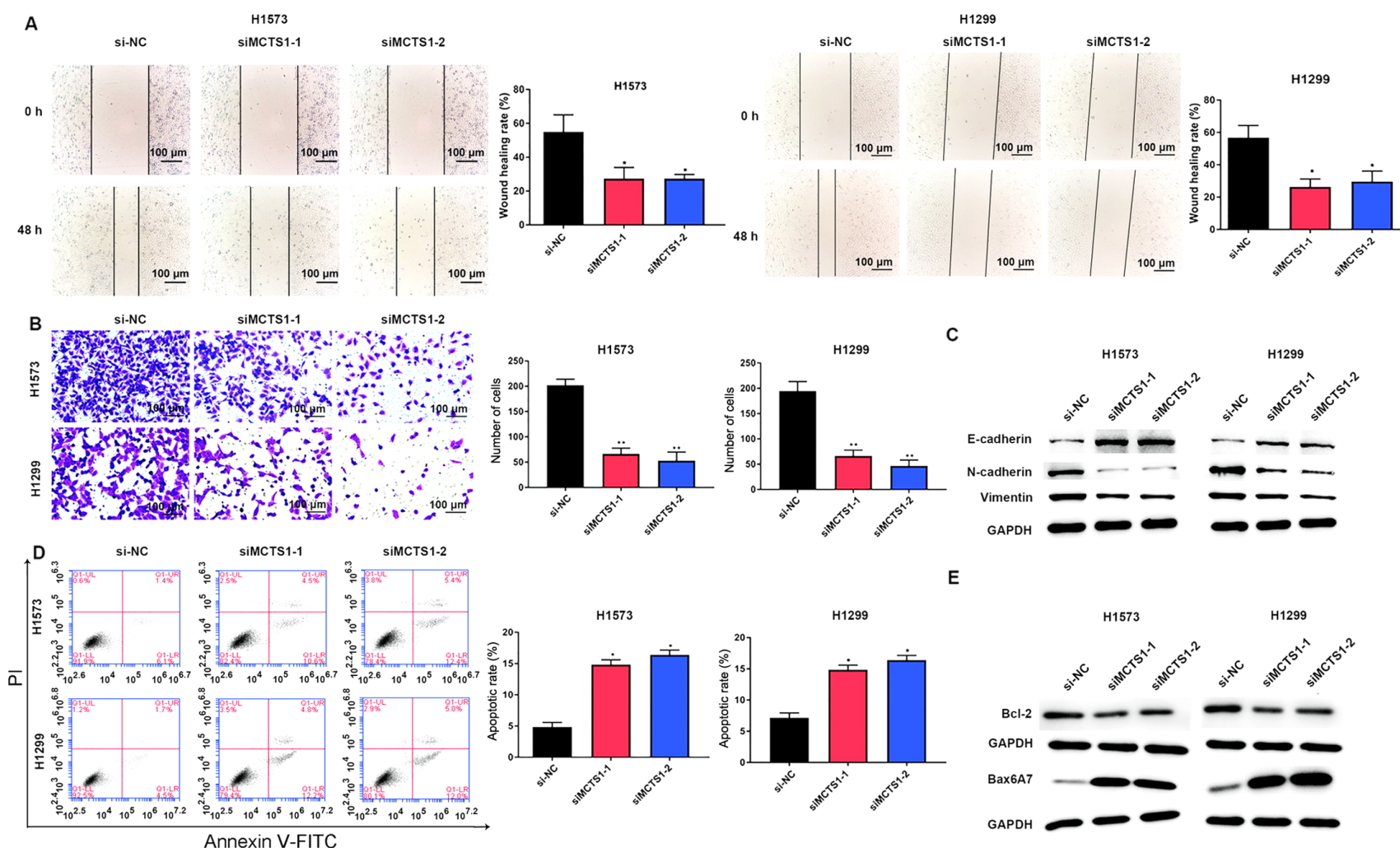

E

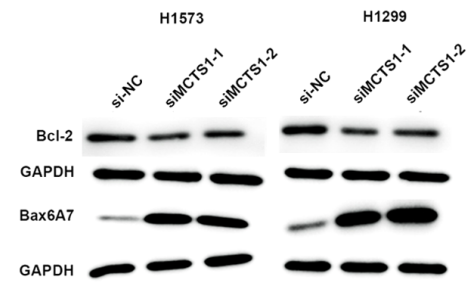

Figure 3. MCTS1 knockdown impairs the migratory ability but promotes the apoptosis of lung adenocarcinoma cells. The migratory ability of H1573 and H1299 cells was assessed via (A) wound healing, (B) Transwell and (C) western blot analyses. (D) Flow cytometry was performed to determine the apoptotic rates of $\mathrm{H} 1573$ and $\mathrm{H} 1299$ cells, the results of which were confirmed via (E) western blot analysis. ${ }^{*} \mathrm{P}<0.05,{ }^{* *} \mathrm{P}<0.01$ vs. si-NC. MCTS1, multiple copies in T-cell lymphoma-1; si, small interfering; NC, negative control.

of the EdU assay indicated that the proportion of proliferative $\mathrm{H} 1573$ and $\mathrm{H} 1299$ cells decreased following transfection with siMCTS1-1/siMCTS1-2, suggesting that the proliferation ability had been impaired (Fig. 2C). The results of the colony formation assay demonstrated that MCTS1 knockdown impaired clonogenicity of LUAD cells (Fig. 2D). Similarly, the wound healing and Transwell assays indicated that MCTS1 knockdown significantly suppressed the migratory and proliferative abilities of LUAD cells (Fig. 3A and B).

The EMT related proteins were investigated, and the results demonstrated that MCTS1 knockdown significantly increased E-cadherin expression, while the expression levels of $\mathrm{N}$-cadherin and Vimentin decreased (Fig. 3C). Flow cytometric analysis indicated that MCTS1 knockdown increased the apoptotic rate of LUAD cells (Fig. 3D). Given that Bcl-2 and Bax are the most representative cellular apoptotic proteins (26), their relative expression levels were detected. The results demonstrated that transfection with siMCTS1-1/siMCTS1-2 inhibited Bcl-2 expression but increased Bax expression (Fig. 3E). Collectively, these results suggest that MCTS1 is closely associated with the proliferation of LUAD cells in vitro.

MCTS1 regulates E2F1 expression. GSEA (version 3.0, http://software.broadinstitute.org/gsea/index.jsp) was performed to determine the potential cellular mechanism of MCTS1 in LUAD, and to identify the pathways and corresponding biomarkers between disease and healthy controls with distinct CIMP status in TCGA cohort. The annotated gene set file (h.all.v7.1.symbols.gmt) was used as the reference gene. $\mathrm{P}<0.05$ was considered to indicate statistical significance. The results demonstrated that MCTS1 was significantly associated with the E2F family (Fig. 4A and B). Notably, a previous study suggested that upregulation of MCTS1 modulates E2F1 expression at the translational level (27). Thus, it was speculated that E2F1 may participate in the regulation of MCTS1 in LUAD. To verify this hypothesis, RT-qPCR and western blot analyses were performed to detect E2F1 expression in LUAD cells. The results demonstrated that transfection with siMCTS1-1/siMCTS1-2 markedly inhibited E2F1 expression in H1573 and H1299 cells (Fig. 4C). In addition, considering the importance of E2F1 in the c-Myc pathway (28-30), c-Myc and cyclin E protein expression were detected in MCTS1-silencing LUAD cells, and the results demonstrated that MCTS1 knockdown attenuated both c-Myc and cyclin E expression (Fig. 4C). Taken together, these results suggest that MCTS1 andE2F1 may be involved in LUAD development by modulating the c-Myc pathway. In addition, transfection with pcDNA3.1-E2F1 significantly promoted MCTS1 mRNA expression in both H1573 and H1299 cells (Fig. 4D).

Overexpression of E2F1 reverses the inhibitory effect of MCTS1 knockdown on LUAD cellular malignant behaviors. To determine whether the oncogenic role of MCTS1 is mediated by E2F1, further functional experiments were performed in H1573 and H1299 cells. Western blot analyses of E2F1 and MCTS1 are presented in both E2F1 overexpression and si-MCTS1 groups in Fig. 5A. The results demonstrated that MCTS1 knockdown decreased E2F1 expression at the translation level. The results of the MTT assay demonstrated that overexpression of E2F1 
A

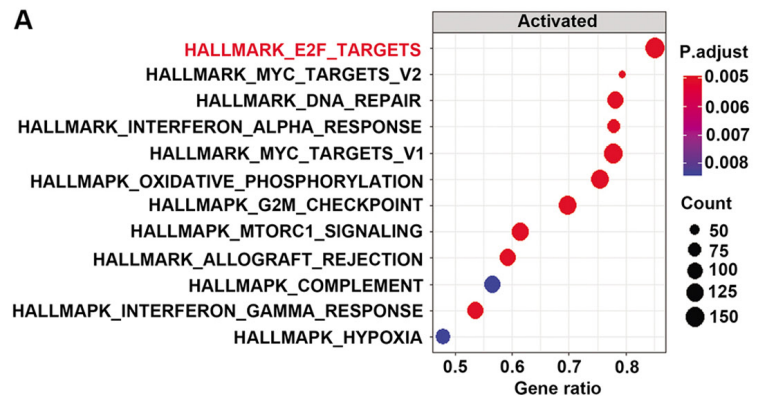

C

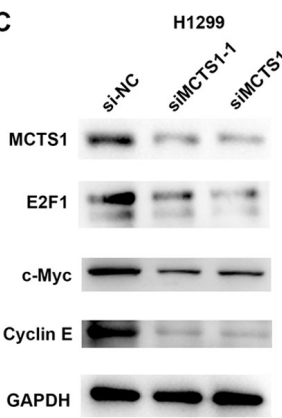

B

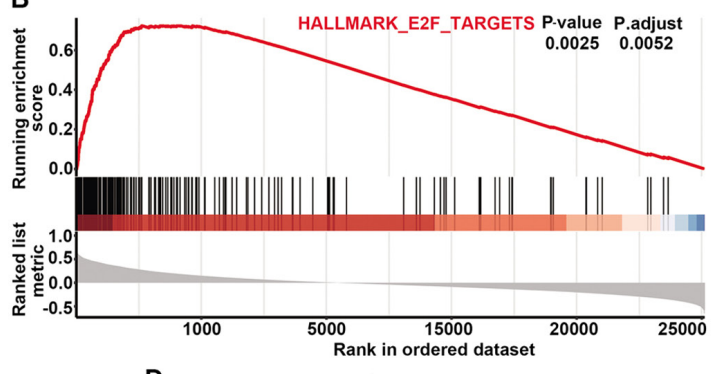

(1)

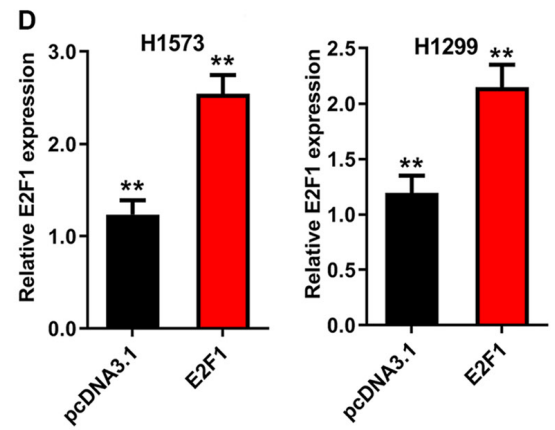

Figure 4. MCTS1 regulates the translation of E2F1. (A and B) Gene set enrichment analysis was performed to predict the pathways of MCTS1 in lung adenocarcinoma. (C) Western blot analysis was performed to detect E2F1, c-Myc and cyclin E expression following MCTS1 knockdown. (D) E2F1 expression significantly increased in $\mathrm{H} 1573$ and $\mathrm{H} 1299$ cells following transfection with pcDNA3.1-E2F1. ${ }^{* *} \mathrm{P}<0.01$ vs. si-NC, si-NC+E2F1, si-NC+pcDNA3.1 or siMCTS1+pcDNA3.1. MCTS1, multiple copies in T-cell lymphoma-1; si, small interfering; NC, negative control.
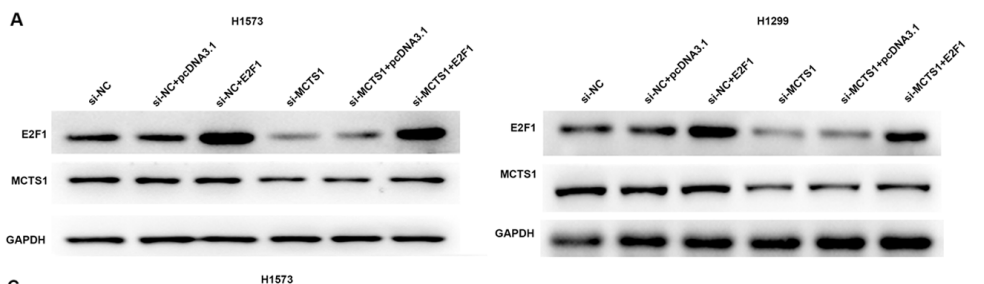

H1573

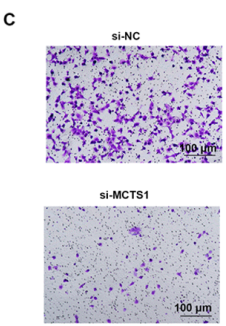

D
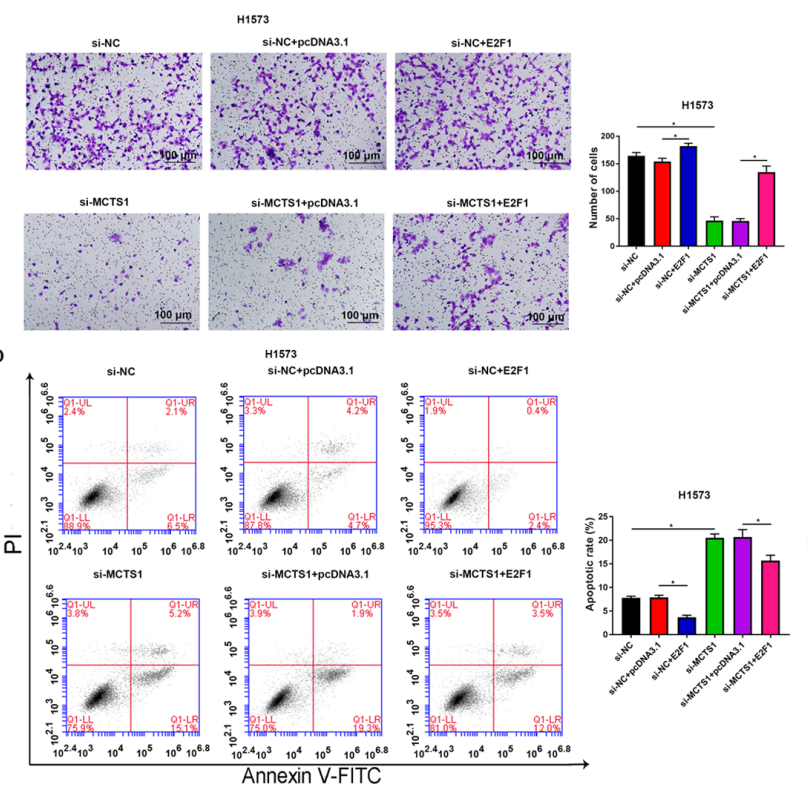

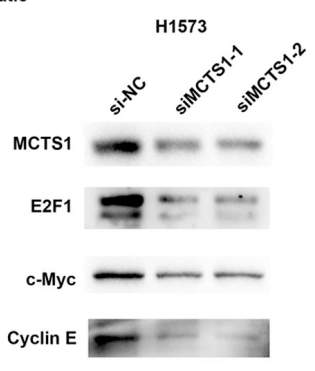

GAPDH
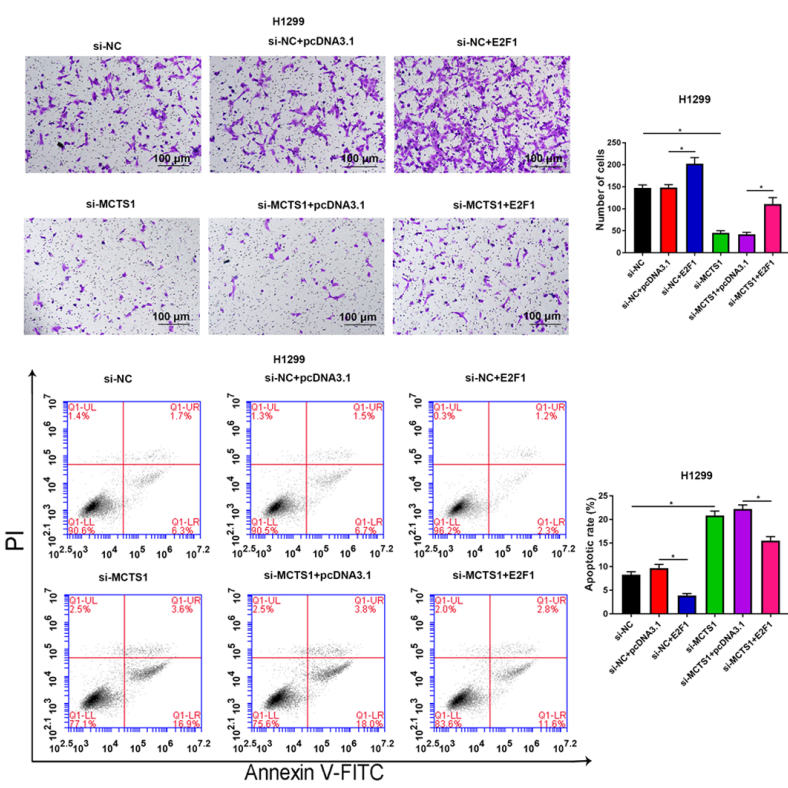

Figure 5. Overexpression of E2F1 reverses the inhibitory effect of MCTS1 knockdown on lung adenocarcinoma cellular malignant behaviors. (A) Western blot analysis was performed to detect the protein expression levels of E2F1 and MCTS1. (B) The MTT assay was performed to assess the proliferation of H1573 and H1299 cells following transfection with si-NC, siMCTS1, siMCTS1+ pcDNA3.1 and siMCTS1+E2F1. (C) Cell migration and (D) apoptosis were investigated following E2F1 knockdown. ${ }^{*} \mathrm{P}<0.05,{ }^{* *} \mathrm{P}<0.01$ vs. si-NC, si-NC+E2F1, si-NC+pcDNA3.1 or siMCTS1+pcDNA3.1. MCTS1, multiple copies in T-cell lymphoma-1; si, small interfering; NC, negative control.

remarkably reversed the anti-proliferation ability of MCTS1 knockdown in H1573 and H1299 cells (Fig. 5B). Consistently, the results of the Transwell assay demonstrated that the suppressive migratory effect of LUAD cells by MCTS1 knockdown 
was reversed following overexpression of E2F1 (Fig. 5C). Flow cytometric analysis demonstrated that the increasing apoptotic rate induced by MCTS1 knockdown was markedly attenuated following overexpression of E2F1 in LUAD cells (Fig. 5D). Collectively, these results suggest that MCTS1 promotes cellular malignant behaviors of LUAD cells and is closely associated with E2F1 regulation.

\section{Discussion}

Lung cancer is the most common cause of cancer-associated mortality worldwide, and LUAD is the most common subtype, accounting for $\sim 40 \%$ of all lung cancer cases $(31,32)$. Increasing evidence suggest that patients with LUAD have detrimental outcomes owing to the lack of effective therapy (33). Thus, it is important to investigate the therapeutic targets for LUAD treatment. Currently, the variants in EGFR, BRAF, and KRAS have been confirmed as the primary pro-cancer candidates in lung cancer $(34,35)$. MCTS1 has received widespread attention due to its involvement in various malignancies, including lung and colon cancers $(36,37)$. Guo et al (28) suggested that MCTS1 and MCTS4 in cancer-endothelial co-culturing microenvironments elevate the proliferation, migration and invasion of renal cancer cells. MCTS1 is the main contributor of lactate uptake in tumor cells and its expression in prostate cancer cells is associated with upregulated LDH-5 expression (38). High MCTS1 expression stimulates cell viability, survival and anchorage-independent growth $(7,39)$. The oncogenicity of MCTS1 is able to overturn the inhibitory effect of p53, and thus continuously accelerate tumorigenesis (40). In addition, MCTS1 is also associated with the stem cell property of A549 cells (8), which suggests the important effect of MCTS1 in lung cancer development. However, to the best of our knowledge, only a few studies have investigated the role of MCTS1 in $\operatorname{LUAD}(8,41)$. The present study analyzed the expression profiles derived from TCGA database and discovered that MCTS1 expression is upregulated in LUAD tissues, which was associated with the clinical stages and metastasis. Furthermore, functional experiments demonstrated that MCTS1 facilitated proliferation and migration, while impairing apoptosis of LUAD cells. Taken together, these results suggest that MCTS1 may promote the progression of LUAD by modulating tumor cell behaviors.

MCTS1 is implicated in multiple molecular mechanisms with broad cellular activity, which has not yet been fully elucidated $(42,43)$. MCTS1 protein can interact with the cap complex and ribosome via the possible RNA-binding motif, a PUA domain, suggesting that MCTS1 may play a crucial role in mRNA translation (44). In addition, over the past decades, several researchers have verified that mRNA translation functions as an essential control point in cell proliferation and differentiation (45). The induction of malignant transformation of cells is associated with the abnormal translation capacity (46). To determine the underlying molecular mechanism of MCTS1 in LUAD, GSEA pathway analyses were performed in the present study. Th e results demonstrated that MCTS1 expression was associated with E2F1. A total of $15 \%$ of all human genes are regulated by the c-Myc protein, and it is well-known that aberrant c-Myc expression is closely associated with tumorigenesis $(28,47)$. E2F1 also participates in the control of c-Myc signals, depending on the oncogenic stress $(48,49)$. Notably, a previous study reported that ectopic MCTS1 expression can promote translational initiation of tumorigenesis-related genes, including E2F1 (27). MCTS1 contains the PUA domain, a RNA-binding domain that can interact with the cap complex through its PUA domain and recruits the density-regulated protein, containing the SUI1 translation initiation domain (27). MCTS1 binds to the cap complex of E2F1 mRNAs and enhance its translation (1). In the present study, RT-qPCR and western blot analyses were performed to determine the transcription and translation relevance between MCTS1 and E2F1 in LUAD. The results demonstrated that MCTS1 knockdown decreased E2F1 expression at the translation level but no effect was observed at the transcription level. Furthermore, rescue experiments demonstrated that the suppressive effect of MCTS1 knockdown on LUAD cellular behaviors was reversed following overexpression of E2F1.

In conclusion, the results of the present study demonstrated that MCTS1 expression was upregulated in LUAD samples, which was associated with poor outcomes in patients with LUAD. Functionally, MCTS1 knockdown attenuated cell proliferation and migration, while inducing apoptosis of LUAD cells. Notably, the anticancer role of MCTS1 knockdown was reversed following overexpression of E2F1. Taken together, these results suggest that MCTS1 may function as a prospective therapeutic target in the management of LUAD treatment by mediating E2F1 and c-Myc signaling pathways.

\section{Acknowledgements}

Not applicable.

\section{Funding}

No funding was received.

\section{Availability of data and materials}

The datasets used and/or analyzed during the current study are available from the corresponding author upon reasonable request.

\section{Authors' contributions}

HT made substantial contributions to conception and design. $\mathrm{CG}$ and RD prepared the experimental materials and performed the experiments. YL, JL and HT interpreted the data, performed the statistical analysis and analyzed the results. CG revised and approved the final version of the manuscript. CG and RD confirm the authenticity of the data. All authors have read and approved the manuscript and agree to be accountable for all aspects of the work in ensuring that the accuracy or integrity of any part of the work are appropriately investigated and resolved.

\section{Ethics approval and consent to participate}

The present study was approved by the Ethics Committee of Qilu Hospital, Cheeloo College of Medicine, Shandong University (Ji'nan, China; approval no. KYLL-2016-097) and written informed consent was provided by all patients prior to the study start. 


\section{Patient consent for publication}

Not applicable.

\section{Competing interests}

The authors declare that they have no competing interests.

\section{References}

1. Siegel RL, Miller KD and Jemal A: Cancer statistics, 2019. CA Cancer J Clin 69: 7-34, 2019.

2. Tan WL, Jain A, Takano A, Newell EW, Iyer NG, Lim WT, Tan EH, Zhai W, Hillmer AM, Tam WL and Tan DSW: Novel therapeutic targets on the horizon for lung cancer. Lancet Oncol 17: e347-e362, 2016.

3. Zhang H, Guo L and Chen J: Rationale for lung adenocarcinoma prevention and drug development based on molecular biology during carcinogenesis. Onco Targets Ther 13: 3085-3091, 2020.

4. Ettinger DS: Ten years of progress in non-small cell lung cancer. J Natl Compr Canc Netw 10: 292-295, 2012.

5. Kim N, Kim HK, Lee K, Hong Y, Cho JH, Choi JW, Lee JI, Suh YL, Ku BM, Eum HH, et al: Single-cell RNA sequencing demonstrates the molecular and cellular reprogramming of metastatic lung adenocarcinoma. Nat Commun 11: 2285, 2020.

6. Mizuno K, Mataki H, Seki N, Kumamoto T, Kamikawaji K and Inoue $\mathrm{H}$ : MicroRNAs in non-small cell lung cancer and idiopathic pulmonary fibrosis. J Hum Genet 62: 57-65, 2017.

7. Prosniak M, Dierov J, Okami K, Tilton B, Jameson B, Sawaya BE and Gartenhaus RB: A novel candidate oncogene, MCT-1, is involved in cell cycle progression. Cancer Res 58: 4233-4237, 1998

8. Li Y, Wang B, Gui S and Ji J: Multiple copies in T-cell malignancy 1 (MCT-1) promotes the stemness of non-small cell lung cancer cells via activating Interleukin-6 (IL-6) signaling through suppressing MiR-34a expression. Med Sci Monit 25: 10198-10204, 2019.

9. Shih HJ, Chen HH, Chen YA, Wu MH, Liou GG, Chang WW, Chen L, Wang LH and Hsu HL: Targeting MCT-1 oncogene inhibits She pathway and xenograft tumorigenicity. Oncotarget 3: 1401-1415, 2012.

10. Wu MH, Chen YA, Chen HH, Chang KW, Chang IS, Wang LH and Hsu HL: MCT-1 expression and PTEN deficiency synergistically promote neoplastic multinucleation through the Src/p190B signaling activation. Oncogene 33: 5109-5120, 2014.

11. Levenson AS, Thurn KE, Simons LA, Veliceasa D, Jarrett J, Osipo C, Jordan VC, Volpert OV, Satcher RL Jr and Gartenhaus RB MCT-1 oncogene contributes to increased in vivo tumorigenicity of MCF7 cells by promotion of angiogenesis and inhibition of apoptosis. Cancer Res 65: 10651-10656, 2005.

12. Dierov J, Prosniak M, Gallia G and Gartenhaus RB: Increased G1 cyclin/cdk activity in cells overexpressing the candidate oncogene, MCT-1. J Cell Biochem 74: 544-550, 1999.

13. Weng YS, Tseng HY, Chen YA, Shen PC, Al Haq AT, Chen LM, Tung YC and Hsu HL: MCT-1/miR-34a/IL-6/IL-6R signaling axis promotes EMT progression, cancer stemness and M2 macrophage polarization in triple-negative breast cancer. Mol Cancer 18: 42, 2019

14. Burkhart DL and Sage J: Cellular mechanisms of tumour suppression by the retinoblastoma gene. Nat Rev Cancer 8: 671-682, 2008

15. Chen HZ, Tsai SY and Leone G: Emerging roles of E2Fs in cancer: An exit from cell cycle control. Nat Rev Cancer 9: 785-797, 2009

16. Wang T, Chen X, Qiao W, Kong L, Sun D and Li Z: Transcription factor E2F1 promotes EMT by regulating ZEB2 in small cell lung cancer. BMC Cancer 17: 719, 2017.

17. Singh S, Yennamalli RM, Gupta $M$ and Changotra $H$ Identification of nsSNPs of transcription factor E2F1 predisposing individuals to lung cancer and head and neck cancer. Mutat Res 821: 111704, 2020

18. Farra R, Grassi G, Tonon F, Abrami M, Grassi M, Pozzato G, Fiotti N, Forte G and Dapas B: The role of the transcription factor E2F1 in hepatocellular carcinoma. Curr Drug Deliv 14: 272-281, 2017

19. Farra R, Dapas B, Grassi M, Benedetti F and Grassi G: E2F1 as a molecular drug target in ovarian cancer. Expert Opin Ther Targets 23: 161-164, 2019
20. Bi XC, Pu XY, Liu JM and Huang S: Effect of transcription factor E2F1 expression on the invasion of prostate cancer. Zhonghua Yi Xue Za Zhi 97: 2856-2859, 2017 (In Chinese).

21. Tsantoulis PK and Gorgoulis VG: Involvement of E2F transcription factor family in cancer. Eur J Cancer 41: 2403-2414, 2005.

22. Yin J, Fu W, Dai L, Jiang Z, Liao H, Chen W, Pan L and Zhao J: ANKRD22 promotes progression of non-small cell lung cancer through transcriptional up-regulation of E2F1. Sci Rep 7: 4430, 2017.

23. Livak KJ and Schmittgen TD: Analysis of relative gene expression data using real-time quantitative PCR and the 2(-Delta Delta C(T)) method. Methods 25: 402-408, 2001.

24. Subramanian A, Tamayo P, Mootha VK, Mukherjee S, Ebert BL, Gillette MA, Paulovich A, Pomeroy SL, Golub TR, Lander ES and Mesirov JP: Gene set enrichment analysis: A knowledge-based approach for interpreting genome-wide expression profiles. Proc Natl Acad Sci USA 102: 15545-15550, 2005.

25. Yu G, Wang LG, Han Y and He QY: clusterProfiler: An R package for comparing biological themes among gene clusters. OMICS 16: 284-287, 2012.

26. Porebska I, Wyrodek E, Kosacka M, Adamiak J, Jankowska R and Harłozińska-Szmyrka A: Apoptotic markers p53, Bcl-2 and Bax in primary lung cancer. In Vivo 20: 599-604, 2006.

27. Reinert LS, Shi B, Nandi S, Mazan-Mamczarz K, Vitolo M, Bachman KE, He H and Gartenhaus RB: MCT-1 protein interacts with the cap complex and modulates messenger RNA translational profiles. Cancer Res 66: 8994-9001, 2006.

28. Guo C, Huang T, Wang QH, Li H, Khanal A, Kang EH, Zhang W, Niu HT, Dong Z and Cao YW: Monocarboxylate transporter 1 and monocarboxylate transporter 4 in cancer-endothelial co-culturing microenvironments promote proliferation, migration, and invasion of renal cancer cells. Cancer Cell Int 19: 170, 2019.

29. Stewart MJ, Litz-Jackson S, Burgess GS, Williamson EA, Leibowitz DS and Boswell HS: Role for E2F1 in p210 BCR-ABL downstream regulation of c-myc transcription initiation. Studies in murine myeloid cells. Leukemia 9: 1499-1507, 1995.

30. Matsumura I, Tanaka H and Kanakura Y: E2F1 and c-Myc in cell growth and death. Cell Cycle 2: 333-338, 2003.

31. Wei S, Zhang ZY, Fu SL, Xie JG, Liu XS, Xu YJ, Zhao JP and Xiong WN: Correction to: Hsa-miR-623 suppresses tumor progression in human lung adenocarcinoma. Cell Death Dis 9: 829, 2018.

32. Xiong DD, Li ZY, Liang L, He RQ, Ma FC, Luo DZ, Hu XH and Chen G: The LncRNA NEAT1 accelerates lung adenocarcinoma deterioration and Binds to Mir-193a-3p as a competitive endogenous RNA. Cell Physiol Biochem 48: 905-918, 2018.

33. Martin LW, D'Cunha J, Wang X, Herzan D, Gu L, Abraham N, Demmy TL, Detterbeck FC, Groth SS, Harpole DH, et al: Detection of occult micrometastases in patients with clinical stage I non-small-cell lung cancer: A prospective analysis of mature results of CALGB 9761 (Alliance). J Clin Oncol 34: 1484-1491, 2016.

34. Ali SA, Justilien V, Jamieson L, Murray NR and Fields AP: Protein Kinase Cı Drives a NOTCH3-dependent Stem-like phenotype in mutant KRAS lung adenocarcinoma. Cancer Cell 29: 367-378, 2016.

35. Calvayrac O, Pradines A, Pons E, Mazières $J$ and Guibert N: Molecular biomarkers for lung adenocarcinoma. Eur Respir J 49: 1601734, 2017.

36. Kasiappan R, Shih HJ, Chu KL, Chen WT, Liu HP, Huang SF, Choy CO, Shu CL, Din R, Chu JS and Hsu HL: Loss of p53 and MCT-1 overexpression synergistically promote chromosome instability and tumorigenicity. Mol Cancer Res 7: 536-548, 2009.

37. Sprowl-Tanio S, Habowski AN, Pate KT, McQuade MM, Wang K, Edwards RA, Grun F, Lyou Y and Waterman ML: Lactate/pyruvate transporter MCT-1 is a direct Wnt target that confers sensitivity to 3-bromopyruvate in colon cancer. Cancer Metab 4: 20, 2016

38. Giatromanolaki A, Koukourakis MI, Koutsopoulos A, Mendrinos S and Sivridis E: The metabolic interactions between tumor cells and tumor-associated stroma (TAS) in prostatic cancer. Cancer Biol Ther 13: 1284-1289, 2012.

39. Shi B, Hsu HL, Evens AM, Gordon LI and Gartenhaus RB: Expression of the candidate MCT-1 oncogene in B- and T-cell lymphoid malignancies. Blood 102: 297-302, 2003.

40. Kasiappan R, Shih HJ, Wu MH, Choy C, Lin TD, Chen L and Hsu HL: The antagonism between MCT-1 and p53 affects the tumorigenic outcomes. Mol Cancer 9: 311, 2010.

41. Huang HK, Lee SY, Huang SF, Lin YS, Chao SC, Huang SF, Lee SC, Cheng TH, Loh SH and Tsai YT: Isoorientin decreases cell migration via decreasing functional activity and molecular expression of proton-linked monocarboxylate transporters in human lung cancer cells. Am J Chin Med 48: 201-222, 2020. 
42. Aravind L and Koonin EV: Novel predicted RNA-binding domains associated with the translation machinery. J Mol Evol 48: 291-302, 1999.

43. Johannsson S, Neumann P and Ficner R: Crystal structure of the human tRNA guanine transglycosylase catalytic subunit QTRT1. Biomolecules 8: 81, 2018.

44. Fleischer TC, Weaver CM, McAfee KJ, Jennings JL and Link AJ: Systematic identification and functional screens of uncharacterized proteins associated with eukaryotic ribosomal complexes. Genes Dev 20: 1294-1307, 2006.

45. Holcik M and Sonenberg N: Translational control in stress and apoptosis. Nat Rev Mol Cell Biol 6: 318-327, 2005.

46. Andersen G, Busso D, Poterszman A, Hwang JR, Wurtz JM, Ripp R, Thierry JC, Egly JM and Moras D: The structure of cyclin $\mathrm{H}$ : Common mode of kinase activation and specific features. EMBO J 16: 958-967, 1997.
47. Dang CV, O'Donnell KA, Zeller KI, Nguyen T, Osthus RC and Li F: The c-Myc target gene network. Semin Cancer Biol 16: 253-264, 2006

48. Bell LA and Ryan KM: Life and death decisions by E2F-1. Cell Death Differ 11: 137-142, 2004

49. Wu Z, Zheng S and Yu Q: The E2F family and the role of E2F1 in apoptosis. Int J Biochem Cell Biol 41: 2389-2397, 2009.

(c) (i) $\ominus$ This work is licensed under a Creative Commons CY AC ND Atribution-NonCommercial-NoDerivatives 4.0 International (CC BY-NC-ND 4.0) License. 